UR-1613

$\mathrm{ER} / 40685 / 951$

\title{
TOP MASS MEASUREMENT AND BOTTOM FRAGMENTATION AT THE LHC *
}

\author{
G. CORCELLA \\ Department of Physics and Astronomy, University of Rochester \\ Rochester, NY 14627, U.S.A.
}

\begin{abstract}
We show some recent HERWIG results related to the top quark mass reconstruction at the LHC and discuss possible improvements for studies of the bottom quark fragmentation function in the top decay.
\end{abstract}

The large amount of $t \bar{t}$ pairs which are expected at the future experiments at the $\mathrm{LHC}^{1}$ will allow precise measurements of the top-quark properties. For the sake of an improved measurement of the top mass $m_{t}$, a new approach has been recently suggested $^{2}$, which consists of using the invariant-mass distributions of $J / \psi+\ell$ pairs, where the $J / \psi$ is produced by the decay of a $b$-flavoured hadron and $\ell$ is the lepton coming from the $W$ produced in the top decay $t \rightarrow b W$. Even though the branching ratio for the process $B \rightarrow J / \psi$ is small, it has been shown that it provides a clean signal at the LHC and about $10^{3}$ well-reconstructed final states are expected in one year of high luminosity. The $m_{J / \psi \ell}$ distributions can then be compared with a template of shapes parametrized by the top mass and $m_{t}$ can be fitted. The expected experimental uncertainty, which is dominated by the statistical error, has been estimated to be $\Delta m_{t} \simeq 1 \mathrm{GeV}$. This is a method which crucially relies on the Monte Carlo description of top production and decay and on the model which is used to simulate the $b$-quark hadronization.

Standard Monte Carlo algorithms ${ }^{3,4}$ simulate multiparton radiation in the soft or collinear approximation and leave regions of the phase space completely empty ('dead zones'). In particular, HERWIG simulates the top decay in the top rest frame. We do not have any soft-gluon radiation from the top quark, while the $b$ quark is allowed to radiate gluons in the 'forward' hemisphere $0<\theta_{g}<\pi / 2$, with $\theta_{g}$ being the emission angle of the soft gluon with respect to the $b$ direction $^{5}$. The following parton cascade satisfies the prescription of angular ordering ${ }^{6}$. The total

*Talk given at DPF 2000, Meeting of the Division of Particle and Fields of the American Physical Society, Columbus, Ohio, U. S. A., 9-12 August 2000. 
energy loss due to gluon radiation is roughly correct, as the collinear singularity $\theta_{g} \simeq 0$ is correctly taken into account; nevertheless problems are expected in the angular distributions, since the $W$ 'backward' hemisphere $\pi / 2<\theta_{g}<\pi$ is a dead zone for the shower.

The new version HERWIG $6.1^{7}$ allows gluon radiation in the backward hemisphere via the implementation of matrix-element corrections $^{8}$ : the dead zone is populated according to the $\mathcal{O}\left(\alpha_{S}\right)$ matrix element ('hard correction') and the parton cascade in the already-populated region is corrected by the use of the exact amplitude any time an emission is the 'hardest so far' ('soft correction') ${ }^{9}$.

We wish to use HERWIG 6.1 to study invariant-mass distributions which are relevant to extract $m_{t}$ at the $\mathrm{LHC}^{10,11}$. For simplicity, we study the spectra $m_{B \ell}$, which must be convoluted with the $B \rightarrow J / \psi$ decay spectra to obtain the $m_{J / \psi \ell}$ distributions. In Fig. 1 we show the $m_{B \ell}$ distributions at the LHC according to HERWIG before (6.0) and after (6.1) matrix-element corrections to top decays, for $m_{t}=175 \mathrm{GeV}$; we observe a shift towards lower values of $m_{B \ell}$ after the inclusion of hard and large-angle gluon radiation. In Table 1 we show results for the average values $\left\langle m_{B \ell}\right\rangle$ for different values of $m_{t}$ and observe that the shift induced by matrixelement corrections is of about $800-900 \mathrm{MeV}$. If we parametrize the relation between $\left\langle m_{B \ell}\right\rangle$ and $m_{t}$ according to a straight line, we find, by means of the least-square method:

$$
\begin{aligned}
& 6.1:\left\langle m_{B \ell}\right\rangle=0.568 m_{t}-6.004 \mathrm{GeV}, \epsilon(\mathrm{GeV})=0.057 \\
& 6.0:\left\langle m_{B \ell}\right\rangle=0.559 m_{t}-3.499 \mathrm{GeV}, \epsilon(\mathrm{GeV})=0.052
\end{aligned}
$$

$\epsilon$ being the mean square deviation in the fit.

Inverting the above relations, we find that the values of $m_{t}$ extracted using the two versions HERWIG 6.0 and 6.1 differ by about $1.5 \mathrm{GeV}$, a value which is larger than the expected uncertainty. This implies that, for an accurate reconstruction of the top mass, the corrections to the top decay must be applied. If we set a cut $m_{B \ell}>50 \mathrm{GeV}$ to reduce the effect of possible backgrounds on the low-mass tails, the impact of matrix-element corrections to top decays is reduced to $1 \mathrm{GeV}$, but it is still competitive with respect to the expected uncertainty ${ }^{11}$.

Table 1. Results for the invariant mass $m_{B \ell}$ at the LHC for different values of $m_{t}$.

\begin{tabular}{lccccc}
\hline$m_{t}$ & $\left\langle m_{B \ell}\right\rangle^{6.1}$ & $\sigma(6.1)$ & $\left\langle m_{B \ell}\right\rangle^{6.0}$ & $\sigma(6.0)$ & $\left\langle m_{B l}\right\rangle^{6.0}-\left\langle m_{B l}\right\rangle^{6.1}$ \\
\hline $171 \mathrm{GeV}$ & $91.13 \mathrm{GeV}$ & $26.57 \mathrm{GeV}$ & $92.02 \mathrm{GeV}$ & $26.24 \mathrm{GeV}$ & $(0.891 \pm 0.038) \mathrm{GeV}$ \\
\hline $173 \mathrm{GeV}$ & $92.42 \mathrm{GeV}$ & $26.90 \mathrm{GeV}$ & $93.26 \mathrm{GeV}$ & $26.59 \mathrm{GeV}$ & $(0.844 \pm 0.038) \mathrm{GeV}$ \\
\hline $175 \mathrm{GeV}$ & $93.54 \mathrm{GeV}$ & $27.29 \mathrm{GeV}$ & $94.38 \mathrm{GeV}$ & $27.02 \mathrm{GeV}$ & $(0.843 \pm 0.039) \mathrm{GeV}$ \\
\hline $177 \mathrm{GeV}$ & $94.61 \mathrm{GeV}$ & $27.66 \mathrm{GeV}$ & $95.46 \mathrm{GeV}$ & $27.33 \mathrm{GeV}$ & $(0.855 \pm 0.039) \mathrm{GeV}$ \\
\hline $179 \mathrm{GeV}$ & $95.72 \mathrm{GeV}$ & $28.04 \mathrm{GeV}$ & $96.51 \mathrm{GeV}$ & $27.67 \mathrm{GeV}$ & $(0.792 \pm 0.040) \mathrm{GeV}$ \\
\hline
\end{tabular}

The next step of the presented analysis is a more detailed study of the $b$ fragmentation in the top decay ${ }^{12}$. Another possible approach to obtain the $m_{B \ell}$ distributions is to perform an exact next-to-leading order calculation of the decay rate $t \rightarrow b W(g)$ and convolute the result with the $b$ fragmentation function, taken from 


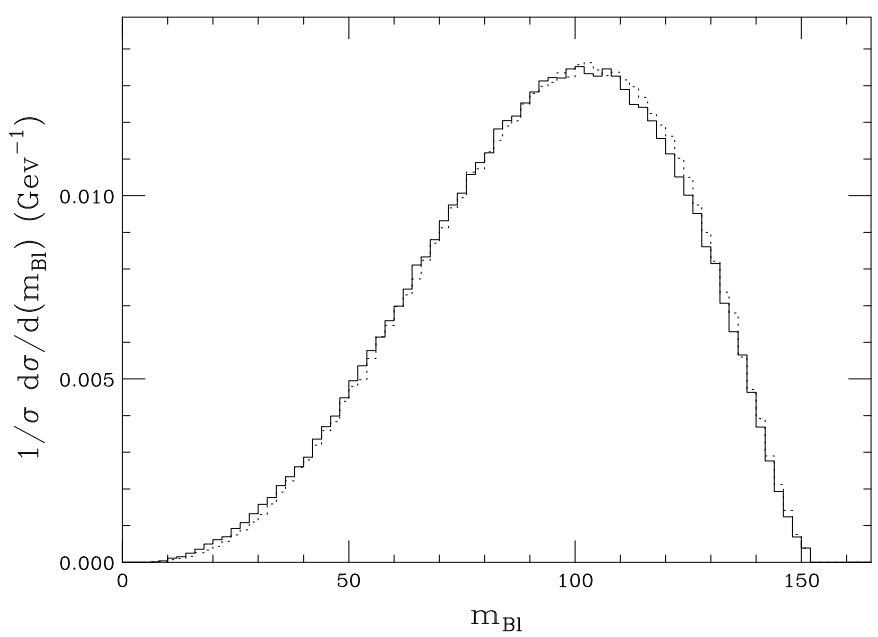

Fig. 1. $m_{B \ell}$ distributions at the LHC according to HERWIG 6.1 (solid histogram) and 6.0 (dotted).

some LEP or SLD data ${ }^{13}$. It will be very interesting to compare the HERWIG results to the ones of this calculation. In order for such a comparison to be trustworthy, we have to tune the parameters of the HERWIG cluster model to fit the $e^{+} e^{-}$data. We have a recent tuning by the OPAL collaboration ${ }^{14}$, after which the agreement of HERWIG to the LEP data is considerably improved. However, even after such a tuning, problems have been found in the comparison to the SLD data ${ }^{15}$, which was not taken into account as an input for the OPAL tuning. The tuning of the HERWIG hadronization model using both LEP and SLD data is in progress ${ }^{12}$.

In summary, we reviewed some HERWIG results which are relevant to the top mass determination at the LHC using final states with leptons and $J / \psi$ and found that the effect of matrix-element corrections to top decays is $\Delta m_{t} \simeq 1.5 \mathrm{GeV}$. We also reported on further studies for a deeper understanding of the $b$ fragmentation in the top decay, using data from the $e^{+} e^{-}$machines.

\section{Acknowledgements}

The presented results have been obtained in collaboration with M.L. Mangano and M.H. Seymour. We acknowledge A. Kharchilava and R. Hemingway for useful discussions and correspondance. This work was supported by grant number DEFG02-91ER40685 from the U.S. Department of Energy.

\section{References}

1. M. Beneke et al., Top Quark Physics, in Proceedings of 1999 CERN Workshop on Standard model physics (and more) at the LHC, G. Altarelli and M.L. Mangano eds., CERN 2000-004, Geneva, Switzerland, 2000, pp. 419-529, hep-ph/0003033.

2. A. Kharchilava, Phys. Lett. B 476 (2000) 73.

3. G. Marchesini et al., Comput. Phys. Commun, 67 (1992) 465. 
4. T. Sjöstrand, Comput. Phys. Commun. 82 (1994) 74.

5. G. Marchesini and B.R. Webber, Nucl. Phys. B 330 (1990) 261.

6. G. Marchesini and B.R. Webber, Nucl. Phys. B 238 (1984) 1.

7. G. Corcella et al., hep-ph/9912396.

8. G. Corcella and M.H. Seymour, Phys. Lett. B 442 (1998) 417.

9. M.H. Seymour, Comput. Phys. Commun. 90 (1995) 95.

10. G. Corcella, J. Phys. G 26 (2000) 634.

11. G. Corcella, M.L. Mangano and M.H. Seymour, JHEP 07 (2000) 004.

12. G. Corcella and A. Mitov, in preparation.

13. SLD collaboration, Phys. Rev. Lett. 84 (2000) 4300.

14. R. Hemingway, OPAL Technical Note TN652.

15. R. Hemingway, private communication. 